\title{
NOTE ON DISTRIBUTIVE POSETS
}

\author{
JAN PASEKA
}

\begin{abstract}
In this note we study distributive posets. We discuss several notions of distributivity in posets and show their equivalence. Moreover, the Prime Ideal Theorem for Distributive Posets is shown to be equivalent with the famous Prime Ideal Theorem for Distributive Lattices using distributive ideal approach.
\end{abstract}

\section{INTRODUCTION}

Distributive ordered sets as well as prime ideals in ordered sets have been studied by many researchers. We shall investigate this notion in the context of the theory of complete Heyting algebras. The paper is organized in the following way. First, we recall basic notions. In Section 1 we discuss the concept of distributivity in ordered sets. We use the complete Heyting algebra of distributive ideals as our main tool to prove both new and old results on distributive posets. In particular, a poset is distributive if and only if any of its cuts is a distributive ideal. The equivalence of the Prime Ideal Theorem for Distributive Posets with the Prime Ideal Theorem for Distributive Lattices and the Representation theorem for Distributive Posets are the main results of Section 2 .

The basic reference for the present text is the classic book by Johnstone [12], where the interested reader can find unexplained terms and notation concerning the subject. We also refer the reader to [2] for standard definitions and notations for lattice structures. For facts concerning the notion of distributivity in posets we refer to papers [4], [5] and [6] by Erné and to [3, 8, 13, 14], for ideals in posets to $[11,15]$. Our terminology and notation agree with the book [12] and the paper [14].

We recall some standard notation. Let $P$ be a poset, $X \subseteq P$. The set

$$
\mathbf{L}(X):=\{y \in P:(\forall x \in X)(y \leq x)\}
$$

is called the left polar of $X$ in $P$. Similarly, the set

$$
\mathbf{U}(X):=\{y \in P:(\forall x \in X)(y \geq x)\}
$$

is called the right polar of $X$ in $P$. If $X=\bigcup_{i=1}^{n} X_{i}$ we put $\mathbf{U}\left(X_{1}, \ldots, X_{n}\right)=\mathbf{U}(X)$ $\left(\mathbf{L}\left(X_{1}, \ldots, X_{n}\right)=\mathbf{L}(X)\right)$. If, moreover, for some $i, 1 \leq i \leq n, X_{i}=\left\{a_{i}\right\}$, we substitute $X_{i}$ by $a_{i}$ as follows $\mathbf{U}\left(X_{1}, \ldots, X_{i}, \ldots, X_{n}\right)=\mathbf{U}\left(X_{1}, \ldots, a_{i}, \ldots, X_{n}\right)$, $\left(\mathbf{L}\left(X_{1}, \ldots, X_{i}, \ldots, X_{n}\right)=\mathbf{L}\left(X_{1}, \ldots, a_{i}, \ldots, X_{n}\right)\right)$.

$M S C$ (2010): primary 06A06, 06A10; secondary 06A11, 06C15, 06C20, $06 \mathrm{D} 99$.

Keywords: distributive poset, distributive ideal, Heyting algebra.

Supported by ESF Project CZ.1.07 /2.3.00/20.0051 Algebraic Methods in Quantum Logic of the Masaryk University. 
Recall that the operators $\mathbf{L}()$ and $\mathbf{U}()$ define a Galois connection on $\mathcal{P}(B)$, i.e., $Y \subseteq \mathbf{L}(X)$ if and only if $X \subseteq \mathbf{U}(Y)$. In particular, $\mathbf{L}()$ and $\mathbf{U}()$ are antitone mappings such that $\mathbf{L}(X)=\bigcap\{\mathbf{L}(x): x \in X\}, \mathbf{U}(Y)=\bigcap\{\mathbf{U}(y): y \in Y\}$, and their compositions $\mathbf{L}() \circ \mathbf{U}()$ and $\mathbf{U}() \circ \mathbf{L}()$ are monotone mappings.

A (Frink) ideal in $P$ is a subset $X \subseteq P$ such that, for each finite subset $Y \subseteq \subseteq X$, we have $\mathbf{L}(\mathbf{U}(Y)) \subseteq X$. A (Frink) filter in $P$ is a subset $X \subseteq P$ such that, for each finite subset $Y \subseteq \subseteq X$, we have $\mathbf{U}(\mathbf{L}(Y)) \subseteq X$. Evidently, the intersection of ideals is an ideal. The least ideal of $P$ containing a subset $X$ of $P$ will be denoted by $\langle X\rangle$. Note that $\langle X\rangle$ is the union of all $\mathbf{L}(\mathbf{U}(A))$ where $A$ runs through the finite subsets of $X$.

A subset $X \subseteq P$ such that $\mathbf{L}(\mathbf{U}(X))=X$ is said to be a cut. The complete lattice of all cuts in $P$ will be denoted by $\mathbf{D M}(P)$. We speak about the DedekindMacNeille completion or completion by cuts.

\section{Distributive Posets}

In what follows, we are going to introduce the notions of a distributive poset and a distributive ideal on it. The complete lattice of distributive ideals is shown to be a complete Heyting algebra. We prefer here the Frink style definition of a distributive poset as in [7] for ideals to the usual ones (see $[16,10,14]$ ).

Definition 2.1. A poset $P$ is said to be distributive if

$$
\mathbf{L}(A, \mathbf{U}(B))=\mathbf{L}(\mathbf{U}(\bigcup\{\mathbf{L}(A, b): b \in B\}))
$$

for all finite subsets $A, B \subseteq \subseteq P$.

Recall that, for $B=\emptyset$, we put $\bigcup\{\mathbf{L}(A, b): b \in B\}:=\emptyset$. The proof of the following lemma is based on an idea from [14, Proposition 31].

Lemma 2.2. Let $P$ be a poset. Then $P$ is distributive iff

$$
\mathbf{L}(a, \mathbf{U}(B))=\mathbf{L}(\mathbf{U}(\bigcup\{\mathbf{L}(a, b): b \in B\}))
$$

for all $a \in P$ and for all finite subsets $B \subseteq \subseteq P$.

Proof. Leftright implication is evident. Let (2.1) hold. If $A=\emptyset$ or $B=\emptyset$, the statement is evident. Let $A \neq \emptyset$ and $B \neq \emptyset$. It is enough to check that

$$
\mathbf{U}(\bigcup\{\mathbf{L}(A, b): b \in B\}) \subseteq \mathbf{U}(\mathbf{L}(A, \mathbf{U}(B))) .
$$

Let $t \in \mathbf{L}(A, \mathbf{U}(B))$ and $s \in \mathbf{U}(\bigcup\{\mathbf{L}(A, b): b \in B\})$. Assume on the contrary that $t \not \leq s$. By (2.1), for all $a \in A, s \notin \mathbf{U}(t)=\mathbf{U}(\mathbf{L}(t))=\mathbf{U}(\mathbf{L}(t, a))$ and $s \notin \mathbf{U}(t)=\mathbf{U}(\mathbf{L}(t, \mathbf{U}(B)))=\mathbf{U}(\bigcup\{\mathbf{L}(t, b): b \in B\})=\bigcap\{\mathbf{U}(\mathbf{L}(t, b)): b \in B\}$. It follows that there is a $b(s, t) \in B$ such that $s \notin \mathbf{U}(\mathbf{L}(t, b(s, t)))$. Hence, there exists a $t_{0} \in \mathbf{L}(t, b(s, t)) \backslash \mathbf{L}(s)$.

Now, let us assume that $A=\left\{a_{1}, \ldots, a_{n}\right\}$. We can construct a finite sequence $t_{1}, \ldots, t_{n}$ by recursion as follows: For $1 \leq i \leq n$, choose $t_{i} \in \mathbf{L}\left(t_{i-1}, a_{i}\right) \backslash \mathbf{L}(s)$. Evidently, $t_{n} \leq t_{n-1} \leq \cdots \leq t_{1} \leq t_{0} \leq b(s, t)$ and $t_{n} \in \mathbf{L}(A) \cap \mathbf{L}(b(s, t))$. This yields $t_{n} \leq s$, a contradiction with $t_{i} \notin \mathbf{L}(s)$ for all $0 \leq i \leq n$.

Hence, $s \in \mathbf{U}(\bigcup\{\mathbf{L}(A, b): b \in B\})$ implies that $s \in \mathbf{U}(\mathbf{L}(A, \mathbf{U}(B)))$. 
Note that the preceding Lemma establishes an equivalence of our definition of a distributive poset with the usual ones (see $[16,10,14])$.

Definition 2.3. A lower subset $I$ of a poset $P$ is said to be a distributive ideal if

$$
\bigcup\{\mathbf{L}(A, b): b \in B\} \subseteq I \text { implies } \mathbf{L}(A, \mathbf{U}(B)) \subseteq I
$$

for all finite subsets $A, B \subseteq \subseteq P$.

Recall that any distributive ideal of a poset is a Frink ideal (putting $A=\emptyset$ in (2.2)). Dually, an upper subset $F$ of a poset $P$ is said to be a distributive filter if

$$
\bigcup\{\mathbf{U}(A, b): b \in B\} \subseteq F \text { implies } \mathbf{U}(A, \mathbf{L}(B)) \subseteq F
$$

for all finite subsets $A, B \subseteq \subseteq P$ and any distributive filter is a Frink filter.

The set of all Frink (distributive) ideals of a poset $P$ will be denoted by $\operatorname{Id}(P)$ $(d-I d(P))$.

Theorem 2.4. Let $P$ be a poset. Then $(d-I d(P), \subseteq)$ with an operation $\Rightarrow$ defined for all $X, Y \subseteq P, X, Y$ lower subsets of $P$, by

$$
Y \Rightarrow X=\{z \in P: \mathbf{L}(z) \cap \mathbf{L}(y) \subseteq X \text { for all } y \in Y\}
$$

is a complete Heyting algebra.

Moreover, the following conditions are equivalent:

(a) $\operatorname{DM}(P) \subseteq d-I d(P)$,

(b) $P$ is a distributive poset.

Proof. Note that $(d-I d(P), \subseteq)$ is a complete lattice - the intersection of any family of distributive ideals is a distributive ideal again.

We have

(1) $Z \subseteq Y \Rightarrow X$ iff $Y \cap Z \subseteq X$ iff $Y \subseteq Z \Rightarrow X$.

(2) If $X$ is a distributive ideal, then so is $Y \Rightarrow X$. Indeed, let $\bigcup\{\mathbf{L}(A, b): b \in$ $B\} \subseteq Y \Rightarrow X, A, B \subseteq \subseteq P$. Then $\bigcup\{\mathbf{L}(A, b): b \in B\} \cap \mathbf{L}(y) \subseteq X$ for all $y \in Y$, i.e., $\bigcup\{\mathbf{L}(A, b, y): b \in B\} \subseteq X$. But $X$ is a distributive ideal. We have $\mathbf{L}(A \cup\{y\}, \mathbf{U}(B))=\mathbf{L}(y) \cap \mathbf{L}(A, \mathbf{U}(B)) \subseteq X$, i.e., $\mathbf{L}(A, \mathbf{U}(B)) \subseteq$ $Y \Rightarrow X$.

It follows that $(d-I d(P), \subseteq)$ with an operation $\Rightarrow$ is a complete Heyting algebra.

Assume first that $P$ is a distributive poset. Let $\mathbf{L}(\mathbf{U}(X))$ be a cut of $P$ and let $A, B \subseteq \subseteq P$. If $\bigcup\{\mathbf{L}(A, b): b \in B\} \subseteq \mathbf{L}(\mathbf{U}(X))$ then $\mathbf{L}(\mathbf{U}(\bigcup\{\mathbf{L}(A, b): b \in B\})) \subseteq$ $\mathbf{L}(\mathbf{U}(X))$, i.e., $\mathbf{L}(A, \mathbf{U}(B)) \subseteq \mathbf{L}(\mathbf{U}(X))$.

Conversely, let us assume that $\mathbf{D M}(P) \subseteq d-I d(P)$. Let $A, B \subseteq \subseteq P$. Evidently, $\bigcup\{\mathbf{L}(A, b): b \in B\} \subseteq \mathbf{L}(\mathbf{U}(\bigcup\{\mathbf{L}(A, b): b \in B\})) \in \mathbf{D M}(P) \subseteq d-I d(P)$. It follows that $\mathbf{L}(A, \mathbf{U}(B)) \subseteq \mathbf{L}(\mathbf{U}(\bigcup\{\mathbf{L}(A, b): b \in B\}))$. The other inclusion is evident.

Lemma 2.5. Let $P$ be a poset. Then $P$ is distributive iff

$$
\mathbf{U}(A, \mathbf{L}(B))=\mathbf{U}(\mathbf{L}(\bigcup\{\mathbf{U}(A, b): b \in B\}))
$$

for all finite subsets $A, B \subseteq \subseteq P$. 
Proof. It is enough to check the leftright implication. The other implication follows by a duality argument. The equality (2.3) is equivalent to the equality

$$
\mathbf{L}(\mathbf{U}(A, \mathbf{L}(B)))=\bigcap\{\mathbf{L}(\mathbf{U}(A, b)): b \in B\}
$$

for all finite subsets $A, B \subseteq \subseteq P$. By the distributivity of the complete Heyting algebra $(d-I d(P), \subseteq)$, we have the second equality below and, by the fact that cuts are in $d-I d(P)$, we get the first inclusion

$$
\begin{aligned}
\bigcap\{\mathbf{L}(\mathbf{U}(A, b)): b \in B\} & =\bigwedge\{\mathbf{L}(\mathbf{U}(A, b)): b \in B\} \\
& =\mathbf{L}(\mathbf{U}(A)) \vee \bigwedge\{\mathbf{L} \mathbf{U}(b): b \in B\}=\mathbf{L}(\mathbf{U}(A)) \vee \mathbf{L}(B) \\
& \subseteq \mathbf{L}(\mathbf{U}(\mathbf{L}(\mathbf{U}(A)), \mathbf{L}(B)))=\mathbf{L}(\mathbf{U}(A, \mathbf{L}(B))) \\
& \subseteq \bigcap\{\mathbf{L}(\mathbf{U}(A, b)): b \in B\} .
\end{aligned}
$$

Remark 2.6. It is well known that a poset $P$ is distributive if and only if

$$
\mathbf{L}(a, \mathbf{U}(b, c))=\mathbf{L}(\mathbf{U}(\mathbf{L}(a, b), \mathbf{L}(a, c)))
$$

for all elements $a, b, c \in P$ (see $[14,10])$.

This is also equivalent to the condition

$$
\mathbf{L}(A, \mathbf{U}(b, c))=\mathbf{L}(\mathbf{U}(\mathbf{L}(A, b), \mathbf{L}(A, c)))
$$

for all finite subsets $A \subseteq \subseteq P$ and for all elements $b, c \in P$.

Now, let us slightly modify Definition 2.3 according to $[16,10]$.

Definition 2.7. A lower subset $I$ of a poset $P$ is said to be

(1) a 2-ideal if

$$
\mathbf{L}(b), \mathbf{L}(c) \subseteq I \text { implies } \mathbf{L}(\mathbf{U}(b, c)) \subseteq I
$$

for all elements $b, c \in P$,

(2) a 2-distributive ideal if

$$
\mathbf{L}(A, b), \mathbf{L}(A, c) \subseteq I \text { implies } \mathbf{L}(A, \mathbf{U}(b, c)) \subseteq I
$$

for all finite subsets $A \subseteq \subseteq P$ and for all elements $b, c \in P$.

Recall that any (distributive) ideal of a poset is a 2-(distributive) ideal. Dually, an upper subset $F$ of a such poset $P$ is said to be

(1) a 2-filter if

$$
\mathbf{U}(b), \mathbf{U}(c) \subseteq F \text { implies } \mathbf{U}(\mathbf{L}(b, c)) \subseteq F
$$

for all elements $b, c \in P$,

(2) a 2-distributive filter if

$$
\mathbf{U}(A, b), \mathbf{U}(A, c) \subseteq F \text { implies } \mathbf{U}(A, \mathbf{L}(b, c)) \subseteq F
$$

for all finite subsets $A \subseteq \subseteq P$ and for all elements $b, c \in P$.

Any (distributive) filter is a 2-(distributive ) filter.

The following is a restatement of Proposition 2.4 and we omit the proof. 
Proposition 2.8. Let $2-d I d(P)$ be the set of all 2-distributive ideals of a poset $P$. Then $(2-d \operatorname{Id}(P), \subseteq)$ is a complete Heyting algebra.

Remark 2.9. Let $2-I d(P)$ be the set of all 2 -ideals of a poset $P$. Note that Halaš in [9] has shown that the distributivity of the complete lattice $2-\operatorname{Id}(P)$ of all 2-ideals of a poset $P$ yields the distributivity of $P$. We are now going to establish the respective statement for the complete lattice $\operatorname{Id}(P)$ of all ideals of $P$.

Theorem 2.10. Let $P$ be a poset and $I d(P)$ a distributive lattice. Then $P$ is distributive.

Proof. It is enough to check Equation 2.4. Let $A, B \subseteq \subseteq P$. Clearly,

$\mathbf{L}(\mathbf{U}(A, \mathbf{L}(B))) \subseteq \bigcap\{\mathbf{L}(\mathbf{U}(A, b)): b \in B\}$.

Let us prove $\mathbf{L}(\mathbf{U}(A, \mathbf{L}(B))) \supseteq \bigcap\{\mathbf{L}(\mathbf{U}(A, b)): b \in B\}$. By the distributivity of the complete lattice $(\operatorname{Id}(P), \subseteq)$ we have

$$
\begin{aligned}
\bigcap\{\mathbf{L}(\mathbf{U}(A, b)): b \in B\} & =\bigwedge\{\langle A \cup\{b\}\rangle: b \in B\}=\bigwedge\{\langle\langle A\rangle \cup\langle\{b\}\rangle\rangle: b \in B\} \\
& =\bigwedge\{\langle A\rangle \vee\langle\{b\}\rangle: b \in B\}=\langle A\rangle \vee \bigwedge\{\langle\{b\}\rangle: b \in B\} \\
& =\langle A\rangle \vee \mathbf{L}(B)=\langle A \cup \mathbf{L}(B)\rangle \subseteq \mathbf{L}(\mathbf{U}(A, \mathbf{L}(B))) .
\end{aligned}
$$

Remark 2.11. Note that, for a poset $P, \operatorname{Id}(P)$ is always a complete algebraic lattice (see [14, Theorem 7]) and $d-I d(P)$ is always a complete Heyting algebra. It is desirable to have some property that these two sets coincide. Clearly, this condition should by Theorem 2.4 always include the distributivity of $P$ (since $\mathbf{D M}(P) \subseteq I d(P)=d-I d(P)$ yields the distributivity of $P)$. Let us propose the following.

Definition 2.12. Let $P$ be a poset. We say that $P$ has property $W(M)$ if, for each finite subset $A \subseteq \subseteq P$, there is a finite subset $W(A) \subseteq \subseteq P \quad(M(A) \subseteq \subseteq P)$ such that $\mathbf{L}(A)=\bigcup_{w \in W(A)} \mathbf{L}(w) \quad\left(\mathbf{U}(A)=\bigcup_{m \in W(A)} \mathbf{U}(m)\right)$.

Recall that the property $\mathrm{M}$ is sometimes called the 2/3-SFP property (see [1]).

Proposition 2.13. Let $P$ be a distributive poset satisfying property $(\mathrm{W})$ (property (M)). Then any Frink ideal (Frink filter) of $P$ is distributive.

Proof. Let $I$ be a Frink ideal, $\bigcup\{\mathbf{L}(A, b): b \in B\} \subseteq I$. Then $\mathbf{L}(A, b)=$ $\bigcup_{w \in W(A, b)} \mathbf{L}(w)$. Let us put $V=\{w \in W(A, b): b \in B\}$. It follows that $\bigcup\{\mathbf{L}(A, b): b \in B\} \subseteq I=\bigcup_{w \in V} \mathbf{L}(w)$, i.e., $V \subseteq I, V$ is finite and, consequently,

$$
\mathbf{L}(A, \mathbf{U}(B))=\mathbf{L}(\mathbf{U}(\bigcup\{\mathbf{L}(A, b): b \in B\}))=\mathbf{L}(\mathbf{U}(V)) \subseteq I .
$$

The remaining part follows by a duality argument.

Theorem 2.14. Let $P$ be a poset satisfying property $(\mathrm{W})$. Then the following conditions are equivalent:

(a) $d-I d(P)=I d(P)$,

(b) $\operatorname{Id}(P)$ is a distributive lattice,

(c) $P$ is a distributive poset. 
Proof. (a) $\Longrightarrow(\mathrm{b})$ : It follows from Theorem 2.4 .

(b) $\Longrightarrow(\mathrm{c})$ : By Theorem 2.10.

$($ c $) \Longrightarrow($ a): A fortiori, as $I d(P) \subseteq d-I d(P)$ by Proposition 2.13.

Remark 2.15. First, note that it was shown in [5] that, for $\wedge$-semilattices, distributivity and ideal distributivity are equivalent properties. This statement follows from our Theorem 2.14 since any $\wedge$-semilattice satisfies property $(\mathrm{W})$.

Second, it is well known that there is a finite distributive poset $P$ such that the lattice $2-\operatorname{Id}(P)$ of all 2 -ideals of $P$ is not distributive (see [11]). Since any finite poset $P$ satisfies property $(\mathrm{W})$, by Theorem $2.14 \operatorname{Id}(P)$ is a distributive lattice. Hence, Halaš's example will not work in our setting. The relevant example that there is an infinite distributive poset $P$ such that the lattice $I d(P)$ of all ideals of $P$ is not distributive is due to Niederle (see [14]).

\section{Prime Ideal Theorem and Representation Theory}

The main goal of this section is to establish a version of Prime Ideal Theorem for distributive posets (satisfying property $(\mathrm{W})$ ) and their representation.

Definition 3.1. A map $f$ between posets $P$ and $Q$ is said to be an LUmorphism if

$$
\mathbf{L}(f(A))=\mathbf{L}(f(\mathbf{U}(\mathbf{L}(A))) \quad \text { and } \quad \mathbf{U}(f(B))=\mathbf{U}(f(\mathbf{L}(\mathbf{U}(B)))
$$

for all finite subsets $A, B \subseteq \subseteq P$. Evidently, any LU-morphism preserves order. We say that $f$ is an $\mathbf{L U}$-embedding (LU-isomorphism) if $f$ is one-to-one (the inverse map to $f$ is an $\mathbf{L} \mathbf{U}$-morphism).

Recall that, for any poset $Q$ and any its subset $P$, we have

$$
\mathbf{L}_{P}(W)=\mathbf{L}(W) \cap P, \quad \mathbf{U}_{P}(Y)=\mathbf{U}(Y) \cap P, \quad \mathbf{L}_{P}(Z)=\mathbf{L}\left(\mathbf{U}\left(\mathbf{L}_{P}(Z)\right)\right) \cap P,
$$

for all subsets $W, Y, Z \subseteq P$.

Lemma 3.2. Let $(Q, \leq)$ be a distributive poset, $P$ a subset of $Q$ such that the natural injection is an $\mathbf{L U}$-embedding. Then $\left(P, \leq_{P}\right)$ is a distributive poset.

Proof. Let $a, b, c$ be elements of $P$. Then

$$
\begin{aligned}
\mathbf{L}\left(\mathbf{U}\left(\mathbf{L}_{P}\left(a, \mathbf{U}_{P}(b, c)\right)\right)\right) & \subseteq \mathbf{L}\left(\mathbf{U}\left(\mathbf{L}_{P}(a)\right)\right) \cap \mathbf{L}\left(\mathbf{U}\left(\mathbf{L}_{P}\left(\mathbf{U}_{P}(b, c)\right)\right)\right) \\
& =\mathbf{L}(a) \cap \mathbf{L}(\mathbf{U}(b, c)) \\
& =\mathbf{L}(\mathbf{U}(\mathbf{L}(a, b), \mathbf{L}(a, c))) \\
& =\mathbf{L}(\mathbf{U}(\mathbf{L}(a, b)) \cap \mathbf{U}(\mathbf{L}(a, c))) \\
& =\mathbf{L}\left(\mathbf{U}\left(\mathbf{L}\left(\mathbf{U}_{P}\left(\mathbf{L}_{P}(a, b)\right)\right)\right) \cap \mathbf{U}\left(\mathbf{L}\left(\mathbf{U}_{P}\left(\mathbf{L}_{P}(a, c)\right)\right)\right)\right) \\
& =\mathbf{L}\left(\mathbf{U}\left(\mathbf{L}\left(\mathbf{U}_{P}\left(\mathbf{L}_{P}(a, b)\right)\right), \mathbf{L}\left(\mathbf{U}_{P}\left(\mathbf{L}_{P}(a, c)\right)\right)\right)\right) \\
& =\mathbf{L}\left(\mathbf{U}\left(\mathbf{L}\left(\mathbf{U}_{P}\left(\mathbf{L}_{P}(a, b)\right) \cap \mathbf{U}_{P}\left(\mathbf{L}_{P}(a, c)\right)\right)\right)\right) \\
& =\mathbf{L}\left(\mathbf{U}_{P}\left(\mathbf{L}_{P}(a, b)\right) \cap \mathbf{U}_{P}\left(\mathbf{L}_{P}(a, c)\right)\right) .
\end{aligned}
$$

This gives us 


$$
\begin{aligned}
\mathbf{L}_{P}\left(a, \mathbf{U}_{P}(b, c)\right) & =\mathbf{L}\left(\mathbf{U}\left(\mathbf{L}_{P}\left(a, \mathbf{U}_{P}(b, c)\right)\right)\right) \cap P \\
& \subseteq \mathbf{L}\left(\mathbf{U}_{P}\left(\mathbf{L}_{P}(a, b)\right) \cap \mathbf{U}_{P}\left(\mathbf{L}_{P}(a, c)\right)\right) \cap P \\
& =\mathbf{L}_{P}\left(\mathbf{U}_{P}\left(\mathbf{L}_{P}(a, b), \mathbf{L}_{P}(a, c)\right)\right) .
\end{aligned}
$$

Proposition 3.3. Let $P$ be a distributive poset. Then the poset

$$
\begin{aligned}
\mathbf{G}_{0}^{1}(P):= & \left(\left\{\mathbf{L}\left(\mathbf{U}\left(X_{1}\right), \ldots, \mathbf{U}\left(X_{n}\right)\right): n \in \mathbb{N}_{+} \&(\forall i)\left(\emptyset \neq X_{i} \subseteq \subseteq P\right)\right\}\right. \\
& \cup\{\mathbf{L}(\mathbf{U}(\emptyset)), \mathbf{L}(\mathbf{U}(P))\}, \subseteq)
\end{aligned}
$$

is a distributive lattice, $Q:=\{\mathbf{L}(x): x \in P\} \cup\{\mathbf{L}(\mathbf{U}(\emptyset)), \mathbf{L}(\mathbf{U}(P))\}$ generates $\mathbf{G}_{0}^{1}(P)$ in the Dedekind-MacNeille completion $\mathbf{D M}(P)$ and the map $\gamma: P \rightarrow$ $\mathbf{G}_{0}^{1}(P), x \mapsto \mathbf{L}(\mathbf{U}(x))$, is an $\mathbf{L} \mathbf{U}$-embedding.

Proof. It is well known (see [14, Proposition 33]) that the set

$$
\mathbf{G}(P):=\left(\left\{\mathbf{L}\left(\mathbf{U}\left(X_{1}\right), \ldots, \mathbf{U}\left(X_{n}\right)\right): n \in \mathbb{N}_{+} \&(\forall i)\left(\emptyset \neq X_{i} \subseteq \subseteq P\right)\right\}, \subseteq\right)
$$

is a distributive lattice. Hence, also $\mathbf{G}_{0}^{1}(P)$ is a distributive lattice and so we only have to check that $\mathbf{L}(\gamma(A))=\mathbf{L}(\gamma(\mathbf{U}(\mathbf{L}(A)))$ and $\mathbf{U}(\gamma(B))=\mathbf{U}(\gamma(\mathbf{L}(\mathbf{U}(B)))$ for all finite subsets $A, B \subseteq \subseteq P$.

Evidently, $\mathbf{L}(\gamma(A)) \supseteq \mathbf{L}(\gamma(\mathbf{U}(\mathbf{L}(A)))$. Now, let $X \in \mathbf{L}(\gamma(A))$. Then $X \in$ $\mathbf{L}(\gamma(a))$ for all $a \in A$, i.e., $X \subseteq \mathbf{L}(a)$ for all $a \in A$, i.e., $X \subseteq \mathbf{L}(A)$, i.e., $\mathbf{U}(X) \supseteq$ $\mathbf{U}(\mathbf{L}(A))$. Now, let $y \in \mathbf{U}(\mathbf{L}(A))$. Then $y \in \mathbf{U}(X)$, i.e., $\mathbf{L}(y) \supseteq X$, i.e., $\gamma(y) \geq X$, i.e., $X \in \mathbf{L}(\gamma(y))$. So we have $X \in \mathbf{L}(\gamma(\mathbf{U}(\mathbf{L}(A)))$. The other equation can be proved dually.

Definition 3.4. Let $P$ be a poset. The trace of a subset $A \subseteq \mathbf{D M}(P)$ is the set $\operatorname{tr}(A):=\{x \in P: \mathbf{L}(\mathbf{U}(x)) \in A\}$.

Lemma 3.5. Let $P$ be a (distributive) poset, $I \in I d(\mathbf{D M}(P))\left(I \in I d\left(\mathbf{G}_{0}^{1}(P)\right)\right)$. Then $\operatorname{tr}(I) \in I d(P)$. Similarly, let $F \in F i l t(\mathbf{D M}(P))\left(F \in F i l t\left(\mathbf{G}_{0}^{1}(P)\right)\right)$. Then $\operatorname{tr}(F) \in F i l t(P)$.

Proof. Evidently, $\operatorname{tr}(I)$ is a lower subset of $P$. Assume that $X \subseteq \subseteq \operatorname{tr}(I)$. Then, for all $x \in X, \mathbf{L}(\mathbf{U}(x)) \in I$, i.e., $\mathbf{L}(\mathbf{U}(X)) \in I$. Therefore, for all $y \in \mathbf{L}(\mathbf{U}(X))$, $\mathbf{L}(\mathbf{U}(y)) \subseteq \mathbf{L}(\mathbf{U}(X))$, i.e., $\mathbf{L}(\mathbf{U}(y)) \in I$, i.e., $y \in \operatorname{tr}(I)$. It follows $\mathbf{L}(\mathbf{U}(X)) \subseteq \operatorname{tr}(I)$.

We have that $\operatorname{tr}(F)$ is an upper subset of $P$. Now, let $X \subseteq \subseteq \operatorname{tr}(F)$. Then, for all $x \in X, \mathbf{L}(\mathbf{U}(x)) \in F$, i.e., $\bigcap_{x \in X} \mathbf{L}(\mathbf{U}(x))=\mathbf{L}(X) \in F$. We have, for all $y \in \mathbf{U}(\mathbf{L}(X)), \mathbf{L}(\mathbf{U}(y)) \supseteq \mathbf{L}(X)$, i.e., $\mathbf{L}(\mathbf{U}(y)) \in F$, i.e., $y \in \operatorname{tr}(F)$. This implies $\mathbf{U}(\mathbf{L}(X)) \subseteq \operatorname{tr}(F)$.

It can be easily checked that any $\wedge$-prime (distributive) ideal $I$, i.e., $I_{1} \cap I_{2} \subseteq$ yields $I_{1} \subseteq I$ or $I_{2} \subseteq I$, is a prime ideal in the usual sense, i.e., its set-theoretic complement $F$ is a filter. Namely, let $A \subseteq \subseteq F$. Then $\mathbf{L}(a) \nsubseteq I I$ for all $a \in A$. By the $\cap$-irreducibility of $I$, we have $\mathbf{L}(A) \nsubseteq I$. Then there is an element $x \in \mathbf{L}(A)$ such that $x \in F$, i.e., $F \supseteq \mathbf{U}(x) \supseteq \mathbf{U}(\mathbf{L}(A))$. We shall denote the set of all prime (distributive) ideals of $P$ by $P t(I d(P))(P t(d-I d(P)))$ and the set of all $\wedge$-prime (distributive) ideals of $P$ by $P s(I d(P))(P s(d-I d(P)))$. Note that, for distributive lattices, all the above sets of "prime ideals" coincide. 
Theorem 3.6. The following are equivalent:

(i) PIT for distributive lattices, i.e., given a distributive lattice $L, a, b \in L$, $a \not \leq b$, there is a prime ideal $I$ of $L$ such that $a \notin I, b \in I$,

(ii) PIT for distributive posets and Frink ideals, i.e., given a distributive poset $P, a, b \in P, a \not \leq b$, there is a prime ideal $I$ of $P$ such that $a \notin I, b \in I$.

Proof. (i) $\Rightarrow$ (ii) Let $P$ be a distributive poset, $a, b \in P, a \not \leq b$. Then $\mathbf{L}(\mathbf{U}(a)) \nsubseteq$ $\mathbf{L}(\mathbf{U}(b))$. Since $\mathbf{G}_{0}^{1}(P)$ is a distributive lattice, there is a prime ideal $I$ in $\mathbf{G}_{0}^{1}(P)$ such that $\mathbf{L}(\mathbf{U}(a)) \notin I, \mathbf{L}(\mathbf{U}(b)) \in I$. Then $F=\mathbf{G}_{0}^{1}(P)-I$ is a filter in $\mathbf{G}_{0}^{1}(P)$. We put $I^{\prime}=\operatorname{tr}(I) \in I d(P), F^{\prime}=\operatorname{tr}(F) \in F i l t(P)$. Then $I^{\prime} \cup F^{\prime}=P, I^{\prime} \cap F^{\prime}=\emptyset$, $a \in F^{\prime}, b \in I^{\prime}$, i.e., $I^{\prime}$ is prime in $P$.

(ii) $\Rightarrow$ (i) Since any distributive lattice is a distributive poset, the implication follows.

Lemma 3.7. Let $P$ be a poset and let $X=P t(I d(P))$ be the set of all prime ideals of $P$. Then the map $\eta: P \rightarrow \mathcal{P}(X)$ defined by

$$
\eta(a)=X_{a}=\{I \in P t(\operatorname{Id}(P)): a \notin I\}
$$

is a LU-morphism of posets. We call $P$ an LU-poset of sets if $\eta$ is an LUembedding.

Proof. Let $A, B \subseteq \subseteq P$. We have $U \in \mathbf{L}(\eta(a): a \in A)$ iff $U \in \mathbf{L}(\eta(a))$ for all $a \in A$ iff, for any prime ideal $I, I \in U$ implies $a \notin I$ for all $a \in A$ iff, for any prime ideal $I, I \in U$ implies $z \notin I$ for all $z \in \mathbf{U}(\mathbf{L}(A))$ iff, for any prime ideal $I, I \in U$ implies $I \in \eta(\mathbf{U}(\mathbf{L}(A)))$ iff $U \in \mathbf{L}(\eta(\mathbf{U}(\mathbf{L}(A))))$.

Similarly, $W \in \mathbf{U}(\eta(b): b \in B)$ iff, $\bigcup\{\eta(b): b \in B\} \subseteq W$ iff, for any prime ideal $I,(I \in \bigcup\{\eta(b): b \in B\}$ implies $I \in W)$ iff, for any prime ideal $I,(\exists b \in B, b \notin I$ implies $I \in W)$ iff, for any prime ideal $I,(\exists z \in L \mathbf{U}(B), z \notin I$ implies $I \in W)$ iff, for any prime ideal $I,(I \in \bigcup\{\eta(z): z \in \mathbf{L}(\mathbf{U}(B))\}$ implies $I \in W)$ iff $W \in$ $\mathbf{U}(\eta(z): z \in \mathbf{L}(\mathbf{U}(B))$.

As in the case of distributive lattices, we would like the map $\eta$ to be an embedding. To do this, we need distributivity of $P$ and an extra assumption - namely the Prime Ideal Theorem for Distributive Lattices. So we can state the following representation theorem (compare [7, Theorem 4, Theorem 5]).

Theorem 3.8. (PIT) Let $P$ be a poset. Then the following are equivalent:

(i) $P$ is distributive,

(ii) given an ideal $J$ of $P$ and an element $a \notin J$, there exists a prime ideal $I$ such that $J \subseteq I$ and $a \notin I$,

(iii) given $a, b \in P$ with $a \not \leq b$, there exists a prime ideal $I$ such that $a \notin I$ and $b \in I$,

(iv) the map $\eta: P \rightarrow \mathcal{P}(\operatorname{Pt}(\operatorname{Id}(P)))$ is an $\mathbf{L U}$-embedding,

(v) $P$ is an $\mathbf{L U}$-poset of sets.

Proof. (i) $\Rightarrow$ (ii) Let $J$ be an ideal, $a \notin J$. We put $\bar{J}$ to be the ideal in $\mathbf{G}_{0}^{1}(P)$ generated by the set $\gamma(J)$. Evidently, $\gamma(a) \notin \bar{J}$. Then, by the Prime Ideal Theorem for Distributive Lattices, there is a prime ideal $\bar{I}$ in $\mathbf{G}_{0}^{1}(P)$ such that $\bar{J} \subseteq \bar{I}$, $\gamma(a) \notin \bar{I}$. Putting $\bar{F}=G(P)-\bar{I}$, we see that $\bar{F}$ is a prime filter in $\mathbf{G}_{0}^{1}(P)$. Now 
we have $J=\operatorname{tr}(\bar{J}) \subseteq \operatorname{tr}(\bar{I})=I, a \notin I, F=\operatorname{tr}(\bar{F})$, i.e., $I$ is an ideal and $F$ is a filter, $I=P-F$.

(iii) $\Rightarrow$ (iv) We can always find a prime ideal that separates different elements, i.e., $\eta$ is one-to-one.

(ii) $\Rightarrow$ (iii), (iv) $\Rightarrow$ (v) $\Rightarrow$ (i) Trivial.

Corollary 3.9. (PIT) Let $P$ be a distributive poset. Then any ideal of $P$ is the intersection of prime ideals.

Corollary 3.10. (PIT) There is a distributive poset $P$ and a prime ideal $I$ of $P$ such that $I$ is not $a \wedge$-prime ideal.

Proof. We know that there is a distributive poset [14] such that its complete lattice of ideals is not distributive. Since any complete lattice $\wedge$-generated by $\wedge$-prime elements is distributive, we obtain our observation.

Corollary 3.11. (AC) There is a distributive poset $P$ and $a \wedge$-irreducible ideal $I$ of $P$ such that $I$ is not a $\wedge$-prime ideal.

Proof. Applying the same arguments as in 3.10 and Theorem 7.1.7 from [1] that $\operatorname{Id}(P)$ is $\wedge$-generated by its $\wedge$-irreducible elements.

Theorem 3.12. The following are equivalent:

(i) PIT for distributive lattices, i.e., given a distributive lattice $L, a, b \in L$, $a \not \leq b$, there is a prime ideal $I$ of $L$ such that $a \notin I, b \in I$,

(ii) PIT for distributive posets satisfying property (W) and Frink ideals, i.e., given a distributive poset $P, a, b \in P, a \not \leq b$, there is a $\wedge$-prime ideal $I$ of $P$ such that $a \notin I, b \in I$,

(ii) PIT for distributive posets satisfying property (W) and distributive ideals, i.e., given a distributive poset $P, a, b \in P, a \not \leq b$, there is a^-prime distributive ideal $I$ of $P$ such that $a \notin I, b \in I$.

Proof. (i) $\Rightarrow$ (ii) Let $P$ be a distributive poset, $a, b \in P, a \not \leq b$. Then $\mathbf{L}(\mathbf{U}(a)) \nsubseteq$ $\mathbf{L}(\mathbf{U}(b))$. Since $I d P=d-I d P$ is an algebraic complete Heyting algebra, there is a $\wedge$-prime ideal $I \in I d P$ such that $\mathbf{L}(\mathbf{U}(a)) \nsubseteq \subseteq I, \mathbf{L}(\mathbf{U}(b)) \subseteq I$. Then $a \notin I, b \in I$. The implication (ii) $\Rightarrow$ (iii) is clear since $I d P=d-I d P$.

(iii) $\Rightarrow$ (i) Since any distributive lattice is a distributive poset satisfying property (W), the implication follows.

Lemma 3.13. Let $P$ be a poset satisfying property $(\mathrm{W})$ and let $X=P s(\operatorname{Id}(P))$ be the set of all $\wedge$-prime ideals of $P$. Then the map $\mu: P \rightarrow \mathcal{P}(X)$ defined by

$$
\mu(a)=X_{a}=\{I \in P s(I d(P)): a \notin I\}
$$

is a LU-morphism of posets.

Proof. The proof proceeds the same way as that of Lemma 3.7.

Theorem 3.14. (PIT) Let $P$ be a poset satisfying property (W). Then the following are equivalent:

(i) $P$ is distributive,

(ii) given an ideal $J$ of $P$ and an element $a \notin J$, there exists a $\wedge$-prime ideal $I$ such that $J \subseteq I$ and $a \notin I$, 
(iii) given $a, b \in P$ with $a \not \leq b$, there exists a $\wedge$-prime ideal I such that $a \notin I$ and $b \in I$,

(iv) the map $\mu: P \rightarrow \mathcal{P}(P s(I d(P)))$ is an LU-embedding,

(v) $P$ is an $\mathbf{L U}$-poset of sets.

Proof. (i) $\Rightarrow$ (ii) Let $J$ be an ideal, $a \notin J$. Then $\mathbf{L}(\mathbf{U}(a)) \nsubseteq I$. Since $I d P=$ $d-I d P$ is an algebraic complete Heyting algebra, there is a $\wedge$-prime ideal $I \in I d P$ such that $\mathbf{L}(\mathbf{U}(a)) \nsubseteq I, J \subseteq I$. Then $a \notin I, J \subseteq I$.

(iii) $\Rightarrow$ (iv) We can always find a $\wedge$-prime ideal that separates different elements, i.e., $\mu$ is one-to-one.

(ii) $\Rightarrow$ (iii), (iv) $\Rightarrow$ (v) $\Rightarrow$ (i) Trivial.

Acknowledgements. We thank the anonymous referee for the very thorough reading and contributions to improve our presentation of the paper.

\section{REFERENCES}

[1] S. Abramsky and A. Jung, Domain theory, in: S. Abramsky, D. M. Gabbay and T. S. E. Maibaum (eds.), Handbook of Logic in Computer Science, vol. 3, Clarendon Press, 1995.

[2] G. Birkhoff, Lattice theory, 3rd ed., American Mathematical Society Colloquium Publications, New York, 1967.

[3] I. Chajda and J. Rachůnek, Forbidden configurations for distributive and modular ordered sets, Order 5 (1989), 407-423.

[4] E. David and M. Erné, Ideal completion and Stone representation of ideal-distributive ordered sets, Topol. Appl. 44 (1992), 95-113.

[5] M. Erné, Distributivgesetze und die Dedekind'sche Schnittvervollständigung, Abh. Braunschweig. Wiss. Ges. 33 (1982), 117-145.

[6] M. Erné, Semidistributivity, prime ideals and the subbase lemma, Rend. Circ. Mat. Palermo, Ser. II XLI (1992), 241-250.

[7] M. Erné, Prime and maximal ideals of partially ordered sets, Math. Slovaca 56 (2006), 1-22.

[8] R. Halaš, Characterization of distributive sets by generalized annihilators, Arch. Math. (Brno) 30 (1994), 25-27.

[9] R. Halaš, Ideals, annihilators and polars in ordered sets, Ph.D. Thesis, Palacký University, Olomouc, 1994.

[10] R. Halaš, Annihilators and ideals in distributive and modular ordered sets, Acta Univ. Palack. Olomuc. Fac. Rerum. Natur. Math. 34 (1995), 31-37.

[11] R. Halaš and J. Rachůnek, Polars and prime ideals in ordered sets, Discuss. Math., Algebra Stoch. Methods 15 (1995), 43-59.

[12] P. T. Johnstone, Stone spaces, Cambridge Univ. Press, Cambridge, 1982.

[13] J. Larmerová and J. Rachůnek, Translations of distributive and modular ordered sets, Acta Univ. Palack. Olomuc. Fac. Rerum. Natur. Math. 27 (1988), 13-23.

[14] J. Niederle, Boolean and Distributive Ordered Sets: Characterization and Representation by Sets, Order 5 (1995), 189-210.

[15] J. Niederle, Ideals in ordered sets, a unifying approach, Rend. Circ. Mat. Palermo 55 (2006), $287-295$.

[16] J. Rachůnek, Translations des ensembles ordonnés, Math. Slovaca 31 (1981), 337-340.

Jan Paseka, Department of Mathematics and Statistics, Faculty of Science, Masaryk University, Kotlářská 2, CZ-611 37 Brno, Czech Republic

e-mail: paseka@math.muni.cz 